\title{
A Numerical Study of Generalized Multiquadric Radial Basis Function Interpolation
}

\author{
Maggie E. Chenoweth \\ Department of Mathematics, Marshall University \\ Advisor: \\ Dr. Scott A. Sarra \\ Department of Mathematics, Marshall University
}

October 13, 2009

\begin{abstract}
This work focuses on the generalized multiquadric (GMQ) radial basis function. The GMQ is derived from the multiquadric (MQ), which is used in radial basis function $(\mathrm{RBF})$ interpolation. This is a relatively new field of research, and many properties of the GMQ are still unknown. Numerical experiments will be performed involving the GMQ, and results will be analyzed to gain further understanding into this type of function.
\end{abstract}

\section{Introduction}

Radial basis function (RBF) methods are part of an emerging field of mathematics. First studied by Roland Hardy, an Iowa State geodesist, in 1968, these methods allow for scattered data to easily be used in computations. This was previously done using polynomial interpolation, but RBF interpolation has been shown to work in some cases where polynomial interpolation has failed [9]. (A function is said to interpolate a set of data points if it passes through those points.) RBF methods are frequently used to represent topographical surfaces as well as other intricate three-dimensional shapes [11].

A radial basis function, $\phi(r)$, is a one variable, continuous function defined for $r \geq 0$ that has been radialized by composition with the Euclidean norm on $\mathbb{R}^{d}$. RBFs may have a free parameter, the shape parameter, denoted by $\epsilon$. If we are given a set of $N$ centers, $x_{1}^{c}, \ldots, x_{N}^{c}$, in $\mathbb{R}^{d}$, an RBF 
interpolant takes the form

$$
s(x)=\sum_{j=1}^{N} \lambda_{j} \phi\left(\left\|x-x_{j}^{c}\right\|_{2}, \epsilon\right) .
$$

The $\lambda_{j}$ coefficients are chosen by interpolation so that

$$
s\left(x_{i}\right)=f_{i}
$$

at a set of points that usually is located at the $N$ centers. This leads to the linear system

$$
B \lambda=f
$$

which will be solved for the MQ (or GMQ) expansion coefficients. $B$ is an $N \times N$ matrix known as the interpolation matrix or system matrix with entries

$$
b_{i j}=\phi\left(\left\|x_{i}^{c}-x_{j}^{c}\right\|_{2}\right), \quad i, j=1, \ldots, N .
$$

Its entries contain the functions representing the approximation space evaluated at the distances between centers. We evaluate the interpolant at $M$ points using (1) by forming an $M \times N$ evaluation matrix $H$ with entries

$$
h_{i j}=\phi\left(\left\|x_{i}-x_{j}^{c}\right\|_{2}\right), \quad i=1, \ldots, M \text { and } j=1, \ldots, N .
$$

The interpolant is then evaluated at the $M$ points to obtain

$$
f_{a}=H B^{-1} f=H \lambda .
$$

The most popular RBF that is used in applications today is the multiquadric (MQ)

$$
\phi(r)=\sqrt{1+\varepsilon^{2} r^{2}}=\left(1+\varepsilon^{2} r^{2}\right)^{1 / 2} .
$$

The properties of the MQ are well-known. However, a related RBF with properties not as well-known is the generalized multiquadric (GMQ)

$$
\phi(r)=\left(1+\varepsilon^{2} r^{2}\right)^{\beta} \quad \beta=\cdots \frac{-3}{2}, \frac{-1}{2}, \frac{1}{2}, \frac{3}{2} \cdots
$$

For $\beta<0$, the GMQ is strictly positive definite, and for $0<\beta<1$, the GMQ is conditionally positive definite of order one. In both cases, the system matrix for the interpolation problem can be shown to be invertible. With $\beta>1$, the GMQ is conditionally positive definite of order $\lceil\beta\rceil$, and to 
show that the system matrix, $B$, is invertible, it is necessary to append low order polynomials to the RBF interpolant [1].

Through extensive numerical experiments, researchers have recently suggested, but not proven, that the GMQ has desirable properties for $\beta$ with non-half-integer powers $[6,13,14]$. This work will focus on the less understood GMQ. A series of numerical experiments will be analyzed to determine if an optimal value for $\beta$ exists.

\section{RBF Interpolation}

As previously mentioned, Hardy is acknowledged for formulating the MQ. While working as a field engineer from 1947 to 1951, he first became interested in stream and ridge lines. In his 1972 paper he writes, "Someone showed me a 'topographic map' that had been 'designed by a statistician' who obviously knew a lot about statistics, but nothing about topography. It was some sort of 'orthogonal polynomial least squares fit of topography to an array of fixed control points on the surface.' Its resemblance to the actual terrain at any point of correspondence between the map and the ground was purely an accidental occurrence, of that I am certain.... A purely statistical approach tends to obscure the need for a more detailed geometric construction of the surface and moves too soon, to an easier, less correct, but more fashionable problem of curve fitting" [3].

Hardy believed that there should exist an interpolation method containing an exact fit of data to a topographical region. After much investigation, he discovered what would later be known as the multiquadric (MQ) (2). Before the MQ, trigonometric and algebraic polynomials were used. Hardy chose the term "multiquadric" because of the quadric surface. Hardy stated that he had considered "multiquadratic," which many people believe is the term, but he decided against it. The MQ is important because it allows for scattered data to be converted into a very accurate fit model of a graph or surface [4].

In 1979, Franke published an article that further supported Hardy's MQ method. Franke's research dealt with comparing various methods to interpolate a test surface. He stated, "The most impressive method in these tests is the multiquadric method of Hardy. It is consistently best or near best in terms of accuracy, and always results in visually pleasant surfaces. Nonetheless a certain skepticism persists because the method has no apparent mathematical basis to explain its efficacy" [2]. Because of Franke's

extensive research concerning the MQ, he is often credited for introducing 
the MQ into the field of mathematical science.

The next significant time in RBF history was in 1986 when Charles Micchelli, an IBM mathematician, developed the theory behind the MQ method. He proved that the system matrix for the MQ method was invertible [8]. Four years later, physicist Edward Kansa first used the MQ method to solve differential equations [5]. In 1992, results [7] from Madych and Nelson showed the spectral convergence rate of MQ interpolation. Since Kansa's discovery, research in RBF methods has rapidly grown, and RBFs are now considered an effective way to solve partial differential equations and meshless methods [14]. In addition to this, the MQ method has been proven to be a generalization of the pseudospectral methods to complex domains and unstructured grids [10].

\section{Numerical Experiments}

Half-integer exponents have been found to work well for values of $\beta$ in the GMQ (2), but we will investigate non-half-integer powers as well.

According to Wang and Liu [13], $\beta=1.03$ was an "optimal" value for $\beta$ for the GMQ. In 2003, Xaio and McCarthy [14] found that $\beta=1.99$ is an "optimal" value. Also, Kansa has recently suggested that the GMQ has desirable outcomes when $\beta=5 / 2$ [6]. However, the behavior of the GMQ method with various $\beta$ and $\epsilon$ is still not well understood. We perform several experiments to see how these values compare to $\beta=1 / 2$ (the MQ) and to draw some conclusions about the GMQ.

We begin by comparing the shape of the graphs of the GMQ with six values for $\beta$ to see how this exponent influences the graph. As shown in Figure 1, the smaller the absolute value of $\beta$ the flatter the curve. All errors are measured in the infinity norm. 


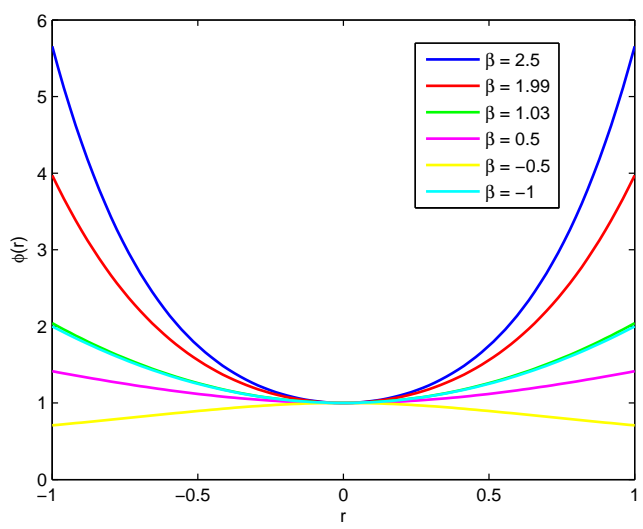

Figure 1: Plot of $\phi(r)$ for six values of $\beta$ when $\epsilon=1$.

\subsection{Experiment 1: Condition Number versus Accuracy}

For our first experiment, we used two different functions

$$
\begin{gathered}
f(x)=e^{x^{3}}+\cos (2 x) \\
f(x)=x^{4}+3 x^{2}-x-2,
\end{gathered}
$$

where $-1 \leq x \leq 1$, to understand how values of $\beta$ affect the accuracy of the RBF method and the condition number of the system matrix. The condition number is a measure of how difficult a problem is to be accurately approximated by a numerical algorithm. In order to well approximate the continuous error, we have evaluated the interpolant at $M=98$ evenly spaced evaluation points We see that when $\beta$ increases, the shape parameter that provides for the best approximation increases as well. In addition to this, the condition numbers increase as the shape parameters decrease. However, there is relatively good accuracy. The results are illustrated in Figures 2, 3, 4 , and 5 . 

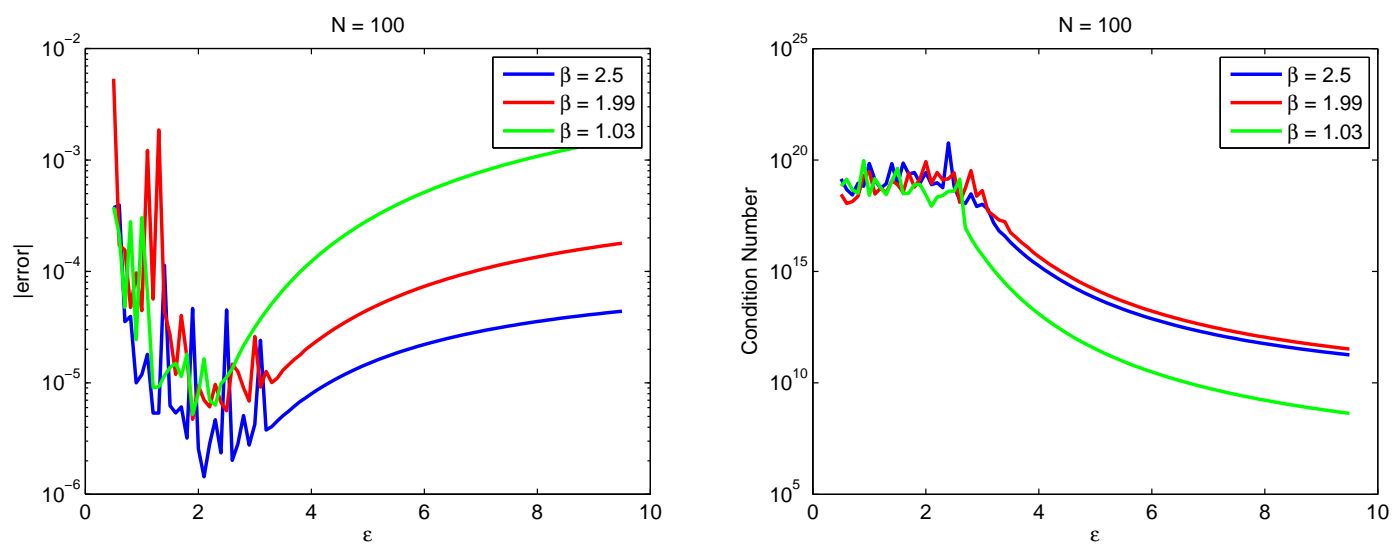

Figure 2: Left: Error for GMQ approximation of test function (4) for values of $\beta>1$. Right: Condition number for GMQ approximation of test function (4) for values of $\beta>1$.
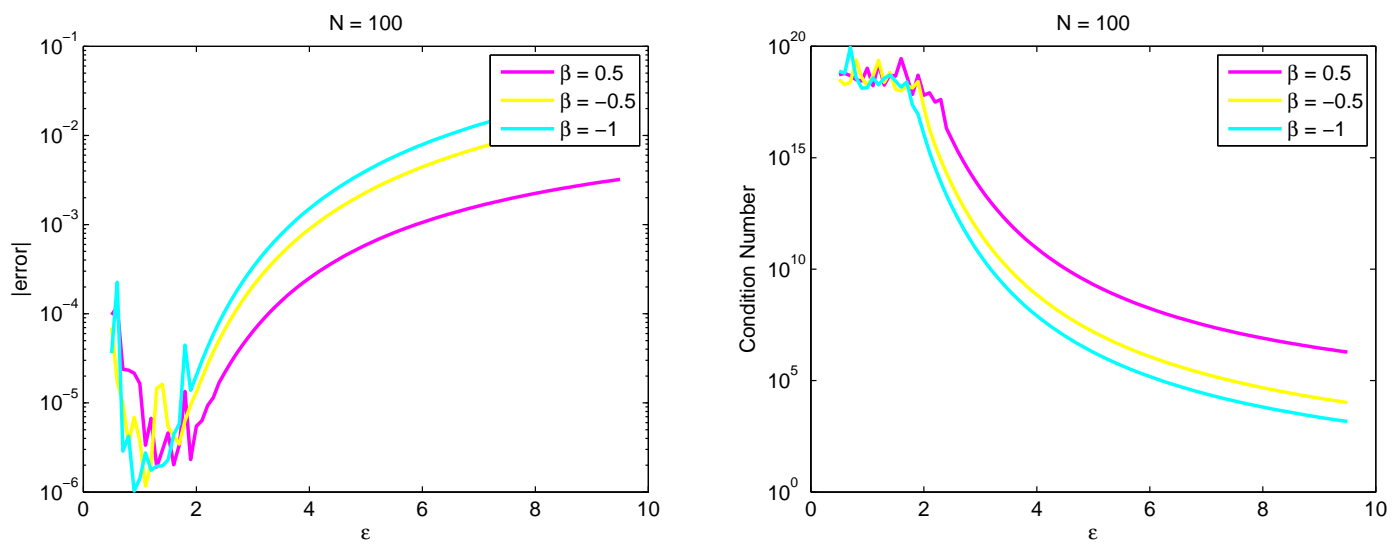

Figure 3: Left: Error for GMQ approximation of test function (4) for values of $\beta<1$. Right: Condition number for GMQ approximation of test function (4) for values of $\beta<1$. 


\begin{tabular}{|c|c|c|}
\hline \multicolumn{3}{|c|}{ TABLE 1: Minimum Errors for Equation (4) } \\
\hline$\beta$ & $\mid$ error $\mid$ & $\epsilon$ \\
\hline 2.5 & $6.9 \mathrm{e}-6$ & 3.8594 \\
1.99 & $9.7 \mathrm{e}-6$ & 3.3295 \\
1.03 & $1.1 \mathrm{e}-5$ & 2.5 \\
0.5 & $9.7 \mathrm{e}-6$ & 2.2465 \\
-0.5 & $7.2 \mathrm{e}-6$ & 1.8318 \\
-1 & $1.3 \mathrm{e}-5$ & 1.8779 \\
\hline
\end{tabular}

Table 1: Comparison between $\beta$, error, and the corresponding shape parameter, $\epsilon$.
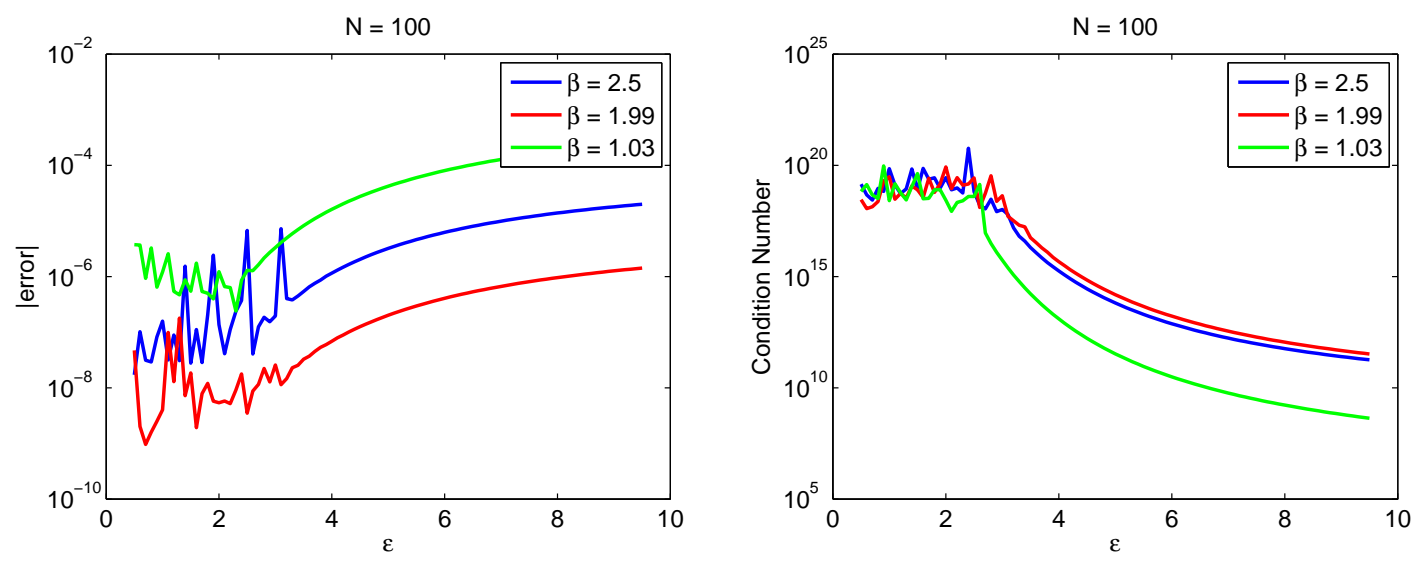

Figure 4: Left: Error for GMQ approximation of test function (5) for values of $\beta>1$. Right: Condition number for GMQ approximation of test function (5) for values of $\beta>1$. 

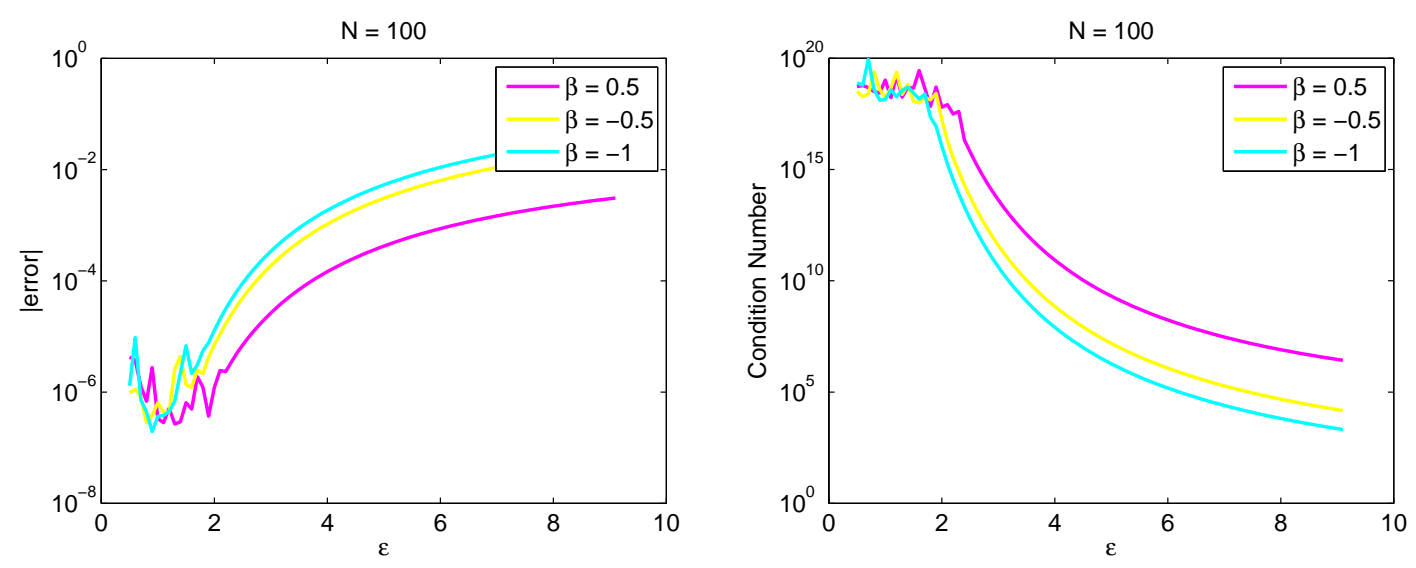

Figure 5: Left: Error for GMQ approximation of test function (5) for values of $\beta<1$. Right: Condition number for GMQ approximation of test function (5) for values of $\beta<1$.

\begin{tabular}{|c|c|c|}
\hline \multicolumn{3}{|c|}{ TABLE 2: Minimum Errors for Equation (5) } \\
\hline$\beta$ & $\mid$ error $\mid$ & $\epsilon$ \\
\hline 2.5 & $4.8 \mathrm{e}-7$ & 3.4687 \\
1.99 & $2.3 \mathrm{e}-8$ & 3.3295 \\
1.03 & $1.2 \mathrm{e}-6$ & 2.6334 \\
0.5 & $2.2 \mathrm{e}-6$ & 2.2235 \\
-0.5 & $2.1 \mathrm{e}-6$ & 1.8318 \\
-1 & $2.0 \mathrm{e}-6$ & 1.5783 \\
\hline
\end{tabular}

Table 2: Comparison between $\beta$, error, and the corresponding shape parameter, $\epsilon$.

Error is measured by taking the infinity norm of the difference of the approximate and exact values for $f(x)$. The minimum error is approximately $10^{-5}$ for all values of $\beta$. Likewise, the condition numbers are similar at the optimal value of $\epsilon$ for each $\beta$. In order for the GMQ system matrix to be wellconditioned, the shape parameter must not be too small. However, small shape parameters are required to obtain good accuracy. Both conditions can obviously not occur at the same time. This is known as the Uncertainty Principle, which indicates the more favorably valued one quantity is the less favorably valued the other is [12]. No optimal value for the shape parameter was found for the set of $\beta$. As $\beta$ increases, the corresponding shape param- 
eter also increases. This was also true when we tested larger values for $\beta$. Our results showed that $\beta=2.5$ provided the best results for function (4), but $\beta=1.99$ provided the best results for function (5). Hence, this indicates that $\beta$ is problem dependent.

\subsection{Experiment 2: Minimum Values for $N$}

Next, for a set of $\beta$, we determine the minimum $N$ needed to obtain the smallest value for the maximum error below a specified tolerance of $10^{-5}$. For this experiment, we used the function

$$
f(x)=e^{\sin (\pi x)}
$$

where $x$ is on the interval $[-1,1]$. Values were obtained by restricting the condition number of the system matrix to be between 1e15 and 1e17. Values $2.5,1.99,1.03,0.5,-0.5$, and -1 for $\beta$ were tested.
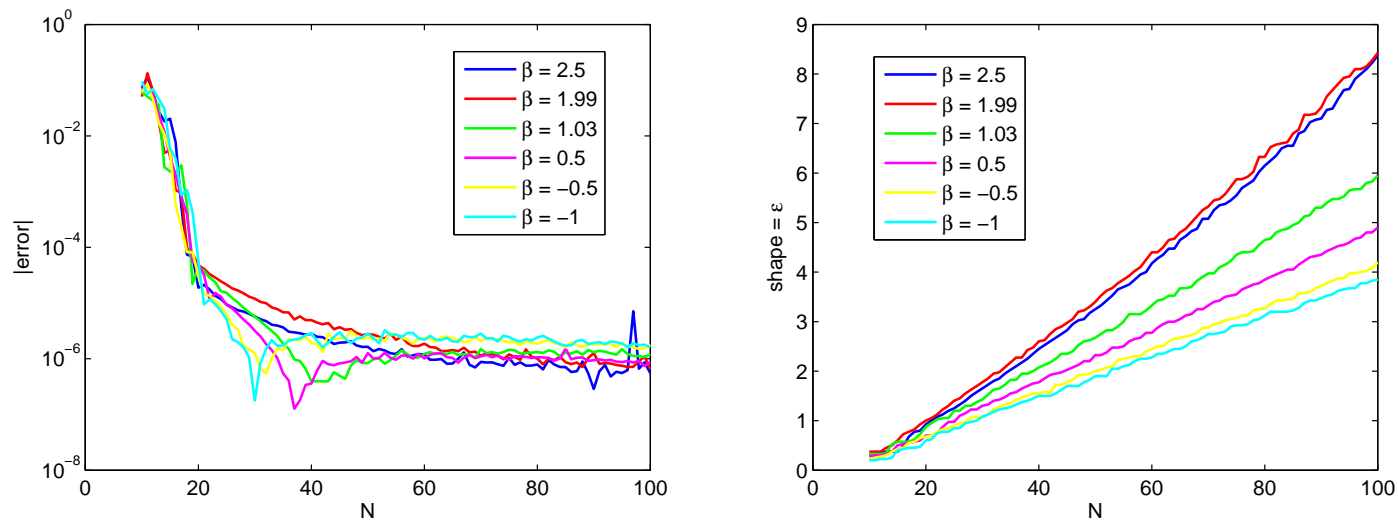

Figure 6: Left: Error for GMQ approximation of test function (6) for values of $\beta$ found by varying the number of $N$ centers. Right: Shape parameter for GMQ approximation of test function (6) for values of $\beta$ found by varying the number of $N$ centers. 


\begin{tabular}{|c|c|c|}
\hline \multicolumn{3}{|c|}{ TABLE 3: $N$ and Shape } \\
\hline$\beta$ & Smallest $N$ & $\epsilon$ \\
\hline 2.5 & 25 & 1.25 \\
1.99 & 32 & 1.94 \\
1.03 & 27 & 1.29 \\
0.5 & 25 & 0.98 \\
-0.5 & 23 & 0.82 \\
-1 & 21 & 0.63 \\
\hline
\end{tabular}

Table 3: Comparison between $\beta$, the number of $N$ centers, and the corresponding shape parameter, $\epsilon$.

From Table 3, we can conclude that there is not a strong relationship between the values of $\beta$ and the corresponding $N$ and $\epsilon$. When $\beta=0.5$ and 2.5 , the smallest number of $N$ centers needed is 25 . A larger variation is in the shape parameter, $\epsilon$.

\subsection{Experiment 3: The Franke Function}

Finally, a two-dimensional example was constructed using the function used by Franke in his test of scattered data approximation methods [2]. Figure 7 is a surface created in Matlab with the GMQ. There are $N=618$ centers and $M=930$ evaluation points. The shape parameter, $\epsilon$, was selected so that the system matrix has a condition number in the range of $1 \mathrm{e} 15$ and 1e17. This range produced the best results in experiment 2 .

\begin{tabular}{|c|c|c|c|}
\hline \multicolumn{4}{|c|}{ TABLE 4: Franke Function } \\
\hline$\beta$ & $\epsilon$ & $\kappa(B)$ & Max Error \\
\hline 2.5 & 4.2 & $5.9 \mathrm{e}+16$ & $7.26 \mathrm{e}-6$ \\
1.99 & 4.5 & $5.3 \mathrm{e}+16$ & $3.14 \mathrm{e}-5$ \\
1.03 & 3.5 & $2.7 \mathrm{e}+16$ & $1.43 \mathrm{e}-5$ \\
0.5 & 3 & $1.1 \mathrm{e}+16$ & $9.23 \mathrm{e}-6$ \\
-0.5 & 2.5 & $2.1 \mathrm{e}+16$ & $8.07 \mathrm{e}-6$ \\
-1 & 2.3 & $3.2 \mathrm{e}+16$ & $8.66 \mathrm{e}-6$ \\
\hline
\end{tabular}

Table 4: Comparison between $\beta$, the shape parameter, the condition number, and the maximum error. 


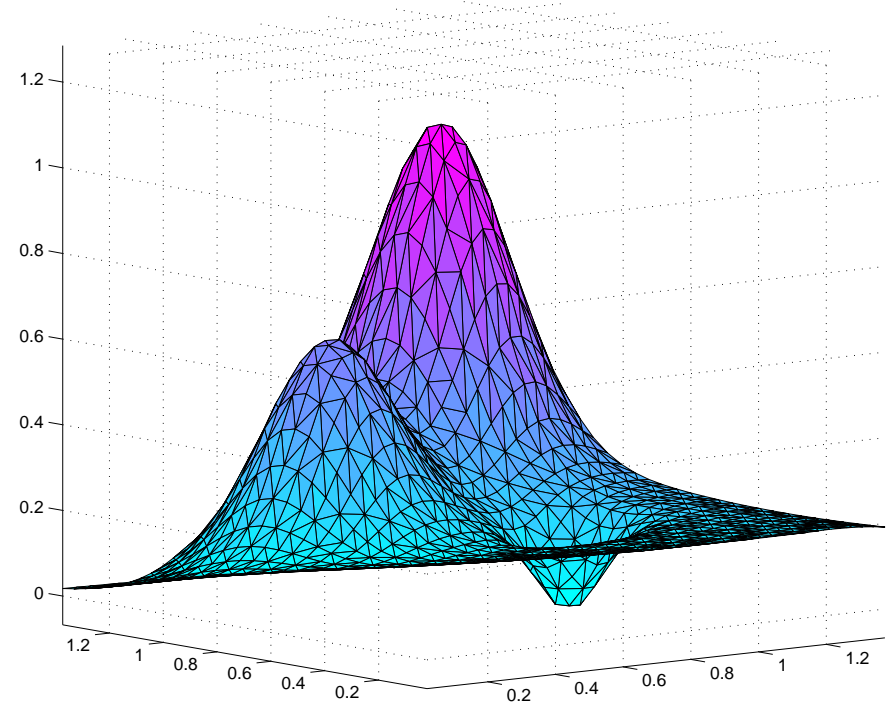

Figure 7: Franke function produced using GMQ interpolation with $\beta=1.03$ and $\epsilon=3$.

\section{Concluding Remarks}

Our numerical experiments suggest that the GMQ interpolation method (which includes the standard MQ when $\beta=1 / 2$ ) produces nearly the same relative accuracy regardless of the value of the exponent $\beta$. The key factor affecting the accuracy of the GMQ method, as dictated by the Uncertainty Principle, is that the system matrix be critically conditioned. We say that the system matrix is critically conditioned when its condition number is such that $1 e 15 \leq \kappa(B) \leq 1 e 17$. Numerical experiments indicate the GMQ method is most accurate when the condition number of the GMQ system matrix is in this range. To obtain a system matrix with a condition number in the critical range, the value of the shape parameter $\varepsilon$ will depend on the value of the exponent $\beta$.

Claims that appeared in the literature stating that certain values of $\beta$ are "optimal" were disproved in our experiments. Instead, $\beta$ seems to be problem dependent, and the suggested optimal values did not produce superior results. 


\section{References}

[1] G. E. Fasshauer. Meshfree Approximation Methods with Matlab. World Scientific, 2007. 1

[2] R. Franke. A critical comparison of some methods for interpolation of scattered data. Technical Report NPS, pages 53-79, 1979. 2, 3.3

[3] R. L. Hardy. Multiquadric equations of topography and other irregular surfaces. Journal of Geophysical Research, 76(8):1905-1915, 1971. 2

[4] R. L. Hardy. Theory and applications of the multiquadric-biharmonic method: 20 years of discovery. Computers and Mathematics with Applications, 19(8), 1990. 2

[5] E. J. Kansa. Multiquadrics - a scattered data approximation scheme with applications to computational fluid dynamics I: Surface approximations and partial derivative estimates. Computers and Mathematics with Applications, 19(8), 1990. 2

[6] E. J. Kansa. Numerical simulation of two-dimensional combustion using mesh-free methods. To appear in Engineering Analysis with Boundary Elements, 2009. 1, 3

[7] W. R. Madych and S. A. Nelson. Bounds on multivariate interpolation and exponential error estimates for multiquadric interpolation. Journal of Approximation Theory, 70:94-114, 1992. 2

[8] C. Micchelli. Interpolation of scattered data: Distance matrices and conditionally positive definite functions. Constructive Approximation, 2:1122, 1986. 2

[9] S. A. Sarra. Radial basis function interpolation. Submitted to SIURO, 2009. 1

[10] S. A. Sarra and E. J. Kansa. Multiquadric Radial Basis Function Approximation Methods for the Numerical Solution of Partial Differential Equations. Tech Science Press, 2009. 2

[11] T. Sauer. Numerical Analysis. Pearson Education, Inc., Boston, 2006. 1

[12] R. Schaback. Error estimates and condition numbers for radial basis function interpolation. Advances in Computational Mathematics, 3:251-264, 1995. 3.1 
[13] J. G. Wang and G. R. Liu. On the optimal shape parameters of radial basis functions used for 2-D meshless methods. Computer Methods in Applied Mechanics and Engineering, 191:2611-2630, 2002. 1, 3

[14] J. R. Xaio and M. A. McCarthy. A local heaviside weighted meshless method for two-dimensional solids using radial basis functions. Computational Mechanics, 31:301-315, 2003. 1, 2, 3 